\title{
Apple Image Segmentation Model Based on R Component with Swarm Intelligence Optimization Algorithm
}

\author{
Liqun Liu ${ }^{\mathrm{a}, *}$ and Jiuyuan Huo ${ }^{\mathrm{b}}$ \\ ${ }^{a}$ College of Information Science and Technology, Gansu Agricultural University, Lanzhou, 730070, China \\ ${ }^{b}$ School of Electronic and Information Engineering, Lanzhou Jiaotong University, Lanzhou, 730070, China
}

\begin{abstract}
Because of large numbers interference factors such as complex background in apple images in natural scene, it is difficult to achieve good image segmentation results. To solve these problems, the color apple image segmentation method under natural scenes is modeled, and an apple image segmentation model based on R component with swarm intelligence optimization algorithm (AISM-RSIOA) is constructed to achieve the initial and secondary segmentation of the images. Under the six conditions of direct sunlight with strong, medium and weak illumination, and backlighting with strong, medium and weak illumination in natural scenes, the images segmentation experiments were taken on a series of mature HuaNiu apple images. The results of initial segmentation showed that the ISMR method has the optimal segmentation effect, and the segmentation success rates achieve $100.0 \%$. In the secondary segmentation stage, the fruits can be fully separated from the background by using the improved threshold segmentation method. The segmentation results demonstrated that the model can effectively improve segmentation effect of images.
\end{abstract}

Keywords: R component; swarm intelligence optimization algorithm; segmentation model; natural scene; apple image

(Submitted on March 11, 2018; Revised on April 21, 2018; Accepted on May 28, 2018)

(C) 2018 Totem Publisher, Inc. All rights reserved.

\section{Introduction}

Apple image recognition is one of the most basic aspects of apple grading [2]. Apple image recognition is used to distinguish apple fruit from the branches and leaves, soil, sky and so on in the background, and it is also denoted as image segmentation [19]. Image segmentation is the basic and critical technology for image processing and computer vision, and it is also the premise of image understanding and pattern recognition [7,23]. In the image segmentation methods, the threshold segmentation method is very important and widely used [16]. The OTSU (Maximal Variance between Classes) method is a classical threshold segmentation method proposed by Japanese scholar OTSU N which uses the grayscale histogram of the image [17]. It dynamically determines the segmentation threshold of image based on the maximum variance between target and background [9,12]. However, it has many defects such as lack of adaptability, easy-to-cause noise and over segmentation [16]. Therefore, the classical OTSU method still needs to further improvement.

Relevant scholars have conducted many studies on identification of apple image from different aspects. The Gaussian mixture global algorithm was proposed for apples recognition by Tabb [22]. The combination method of R-G (Red and Green) and chromatic aberration ratio was used by Si [21] to identify the red apples under different conditions along the front-lighting, backlighting, etc. Zhang [26] took the color features (RGB values) and the texture features as input nodes to build a neural network for apple image segmentation. Wang extracted the color features of apple images and adopted the Support Vector Machine (SVM) for apple image recognition [25]. The results showed that this method is better than the neural network.

\footnotetext{
* Corresponding author.

E-mail address: liulq@gsau.edu.cn
} 
As can be seen from the above research progress, the research conducted on apple image recognition has changed from the artificial background to the natural background, from single color feature to the combination of color and texture features, and from single threshold segmentation to the artificial intelligence direction [1,3,19]. Many scholars have focused on overcoming the defects of the classical OTSU method in searching the optimal threshold. An improved remote-sensing image segmentation method based on improved OTSU and Shuffled Frog-Leaping Algorithm (SFLA) was proposed by Lu [16]. Compared with traditional OTSU image segmentation algorithm and the 2-D OTSU image segmentation algorithm, the experiment results showed that the proposed approach could eliminate the noise interference more effectively and with higher efficiency. A new OTSU method optimized by the Genetic Algorithm (GA) was presented by Li [12]. The experiment result showed that the fuzziness and the noises can be eliminated efficiently. An improved adaptive genetic algorithm (IAGA) was proposed by Jiang [9]. The experiment result showed that the new method can obviously advance the real-time and produce clearer segmented images. These research works show that the combination of the threshold segmentation method and artificial intelligence could greatly improve the image segmentation effect.

In the practical applications, apple images captured in natural scene are more complex, with a large number of branches, leaves and other interfering factors in the background. If the threshold segmentation method or the RGB chromatic aberration ratio method is used, then the segmentation result is not ideal. To solve the above problems, a unified segmentation model should be designed for the apple image segmentation method in natural scene for the segmentation of mature apple images under different scenes and different lighting conditions effectively and accurately.

In this paper, an improved color image cone segmentation method with R component (ISMR) is proposed, an idea gained from the improved RGB color method in literature [8,27]. Then, an apple image segmentation model based on $\mathrm{R}$ component with swarm intelligence optimization algorithm (AISM-RSIOA) is constructed. In the AISM-RSIOA model, the swarm intelligence optimization algorithm is introduced to optimize the threshold of image to overcome the defects of over segmentation in the classical threshold segmentation method. A series of mature HuaNiu apple images under the six light conditions in natural scene were taken for the segmentation experiments.

\section{An Improved Color Image Cone Segmentation Method with R Component (ISMR)}

The color image Cone Segmentation algorithm based on G-component was built on the RGB cube space model. A quartercone was made with the center axis of the concentric G-axis in space, and then the pixels in the inner cone are taken as a target and other pixels are taken as the background [27]. On the basis of the literature [8,27], this paper proposes an improved color image cone segmentation method with R component (ISMR), and improves the parameter $k$ in the method.

As shown in Figure 1, in the RGB cube space model, the intersection points of the cube on the three coordinate axes of $\mathrm{R}, \mathrm{G}$ and $\mathrm{B}$ are set as A, D and E, respectively. Taking the coordinate origin $\mathrm{O}(0,0,0)$ as the vertex, the R coordinate as the center axis, and $\mathrm{r}$ as the bottom radius, a quarter cone was constructed. Then, the conical bottom surface intersects the cube plane parallel to the $\mathrm{G}$-axis to form a circular arc $\stackrel{\cap}{F}$. Taking a point $\mathrm{B}(\mathrm{R}, \mathrm{G}, \mathrm{B})$ on the arc $F G$, the vertical line from point $\mathrm{B}$ intersects the line segment $\mathrm{AG}$ at point $\mathrm{C}$. To make $\angle A O B=\theta$, then the equation is established in Equation (1).

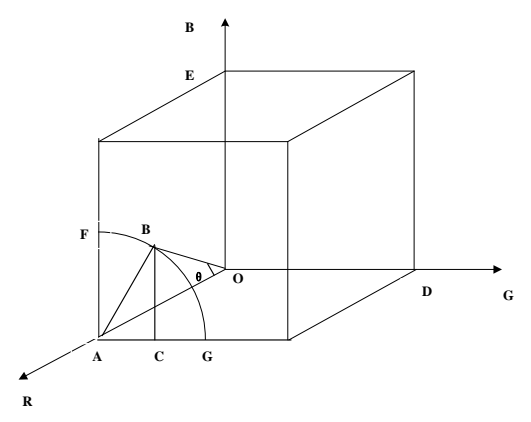

Figure 1. RGB cube space model based on color image cone segmentation with R component

$$
A B=r, A O=R, A C=G, B C=B \text {, and } \angle O A B=\angle A C B=90^{\circ}
$$


In the triangle $\triangle O A B, \operatorname{tg} \theta=\frac{A B}{A O}=\frac{r}{R}$. To make $\operatorname{tg} \theta=k$, therefore $r=R \cdot k$.

In the triangle $\triangle A C B, A B^{2}=A C^{2}+B C^{2}$, that is $r^{2}=G^{2}+B^{2}=R^{2} \cdot k^{2}$, therefore

$$
R^{2}=\frac{G^{2}+B^{2}}{k^{2}}
$$

In accordance with the theory of color image cone segmentation algorithm based on G-component, the pixels in inner cone are taken as a target, and other pixels are taken as the background [27]. Thus, the target pixels are located on the conical surface or inside the conical surface $\hat{O F} G$ in Figure 1. The color image cone segmentation method with $\mathrm{R}$ component is expressed in Equation (2).

$$
g=\left\{\begin{array}{cc}
255, & R^{2} \geq \frac{G^{2}+B^{2}}{k^{2}} \\
0, & \text { others }
\end{array}\right.
$$

Since the value of $\mathrm{k}$ of the color image cone segmentation algorithm based on G-component came from the statistical empirical value, there exists a deviation in the solution which results in a poor segmentation result. The concept of global sharing factor $\alpha_{G}$ was proposed in literature [15]. It is a sharing factor that changes nonlinearly only along with the number of experiments $t$ [24], and it increases rapidly from a small initial value to a steady-state value.

As shown in Equation (3), the number of trials will be applied to the global share factor in this paper.

$$
\begin{gathered}
\alpha_{G}=\left(1-\delta_{G}\right) \cdot \alpha_{\text {final }} \\
\delta_{G}=1-\left(\alpha_{\text {init }} / \alpha_{\text {final }}\right)^{1 / t}, t \in[1, \text { Maxgen }]
\end{gathered}
$$

$\alpha_{\mathrm{G}}$ is the global sharing factor, $\alpha_{\text {init }}$ is the initial value, $\alpha_{\text {final }}$ is the final value, $t$ is the number of global evolution.

Then, the global sharing factor is introduced into Equation (2) to improve the color image cone segmentation method with $\mathrm{R}$ component. The final equation is described in Equation (4).

$$
g=\left\{\begin{array}{cc}
0, & R^{2} \geq \frac{G^{2}+B^{2}}{\alpha_{G}} \\
255-\left(\alpha_{G} \cdot R-G-B\right), \alpha_{G} \cdot R-G-B<255 & \text { others } \\
255, &
\end{array}\right.
$$

\section{An Improved threshold segmentation method}

After the segmentation of the apple image by using the ISMR method, some backgrounds will still be segmented as the targets, which results in extra noise in the segmentation result. In order to solve these problems, this paper adopts the combination of the Swarm Intelligence Optimization Algorithm (SIOA) [11] and ISMR method to segment the apple image. Then, the threshold of the segmented apple image is optimized to choose the most suitable segmentation threshold.

\subsection{Fruit Fly Optimization Algorithm with Fast Global Convergence (FGCFOA)}

The Swarm Intelligence Optimization Algorithm (SIOA) [11] is a kind of heuristic algorithm currently used to solve complex optimization problems [20]. It is an intelligent search algorithm that simulates the natural ecosystem based on the foraging behavior of the species living in the nature. At present, the representative swarm intelligence algorithms mainly include Fruit Fly Optimization Algorithm (FOA) [18], Shuffled Frog Leaping Algorithm (SFLA) [4], Harmony Search Algorithm, HSA) [5] and Artificial Bee Colony Algorithm (ABCA) [10]. 
In this paper, a new Fruit Fly Optimization Algorithm with Fast Global Convergence (FGCFOA) is proposed by applying the global sharing factor mentioned in Section 2. The global sharing factor is applied into the position updating process of fruit fly individual after one time optimization to achieve fast global convergence [6,13]. The position updating of individual flies after one time optimization of FGCFOA is described as follows:

$$
\begin{gathered}
X_{i}=\alpha_{\mathrm{G}} \times X_{i}+X \_ \text {axis }+ \text { RandomValue } \\
Y_{i}=\alpha_{\mathrm{G}} \times Y_{i}+Y_{-} \text {axis }+ \text { RandomValue }
\end{gathered}
$$

The process of FGCFOA is summarized as follows:

Step 1: Initialize the population size Sizepop and maximum iteration number Maxgen , and randomly initialize the population position ( $X_{-}$axis,$Y_{-}$axis ) of fruit flies.

Step 2: At first, the fruit fly individuals randomly search for food using their olfactory organs. Randomly assigning the direction and distance of fruit fly individuals searching for food according to Equation (6), the search distance is expressed as RandomValue.

$$
\begin{gathered}
X_{i}=X \_ \text {_axis }+ \text { Random Value } \\
Y_{i}=Y \_ \text {axis }+ \text { RandomValue }
\end{gathered}
$$

Step 3: Due to the random search mechanism for food, the position of the food cannot be determined. Thus, according to Equation (7), the distance ${ }^{\text {Dist }}{ }_{i}$ between the fruit fly individual and the origin point is calculated first, and then the taste concentration determination value $S_{i}$ of fruit fly individual is calculated by Equation (8).

$$
\begin{aligned}
\text { Dist }_{i} & =\sqrt{X^{2}{ }_{i}+Y_{i}^{2}} \\
S_{i} & =1 / \text { Dist }_{i}
\end{aligned}
$$

Step 4: The taste concentration determination value $S_{i}$ is substituted into the taste concentration determination function (i.e. fitness function) according to Equation (9) to calculate the taste concentration $S m e l l_{i}$ of the fruit fly individual.

$$
\operatorname{Smell}_{i}=\text { Function }\left(S_{i}\right)
$$

Step 5: According to Equation (10), the fruit fly individual with the best taste in the fruit fly population is found (suitable for solving the minimization problem).

$$
[\text { bestSmell bestindex }]=\min \left(\operatorname{Smell}_{i}\right) \operatorname{Smell}_{i}=\operatorname{Function}\left(S_{i}\right)
$$

Step 6: According to Equation (11), the best taste concentration value bestSmell and its X-axis and Y-axis coordinate values are stored, and then other groups of flies will fly to this position by vision.

$$
\begin{gathered}
\text { Smellbest }=\text { best Smell } \\
X \_ \text {axis }=X(\text { bestindex }) \\
Y \_a x i s=Y(\text { bestindex })
\end{gathered}
$$

Step 7: According to Equation (3), the global sharing factor $\alpha_{G}$ is calculated. 
Step 8: According to Equation (5), the position of the fruit fly individual is updated again.

Step 9: The iterative optimization process starts and Step 3 to Step 5 are repeated. If bestSmell $_{\text {new }}<$ bestSmell old $_{\text {is }}$ satisfied, go to Step 6 to Step 8 to continue iterative optimization. Otherwise, go to Step 7 to Step 8 to update the positions of fruit fly individuals and continue the iterative optimization. The above processes are repeated until the maximum number of iterations Maxgen is reached; then, the optimal taste concentration value bestSmell will be outputted.

\subsection{Improved Threshold Segmentation Method}

The improved method of threshold segmentation is realized by the combination of the swarm intelligence algorithm and the classical threshold segmentation method. The above four kinds of swarm intelligence algorithms are used to improve the threshold segmentation method, and then the four improved methods, FGCFOAOTSU, GSF ${ }^{2}$ LAOTSU [15], GSFHSAOTSU [14] and ABCAOTSU, are obtained. The execution flow is showed in Figure 2. The improved method first calculates the gray scale pixels of the target and the background in the apple image, and then calculates the variance between them. The variance between image classes is chosen as the fitness of the swarm intelligence algorithm, and then, the segmentation threshold of apple image is optimized according to the four swarm intelligence algorithm optimization strategies. Finally, the obtained threshold is used for the segmentation of apple image.

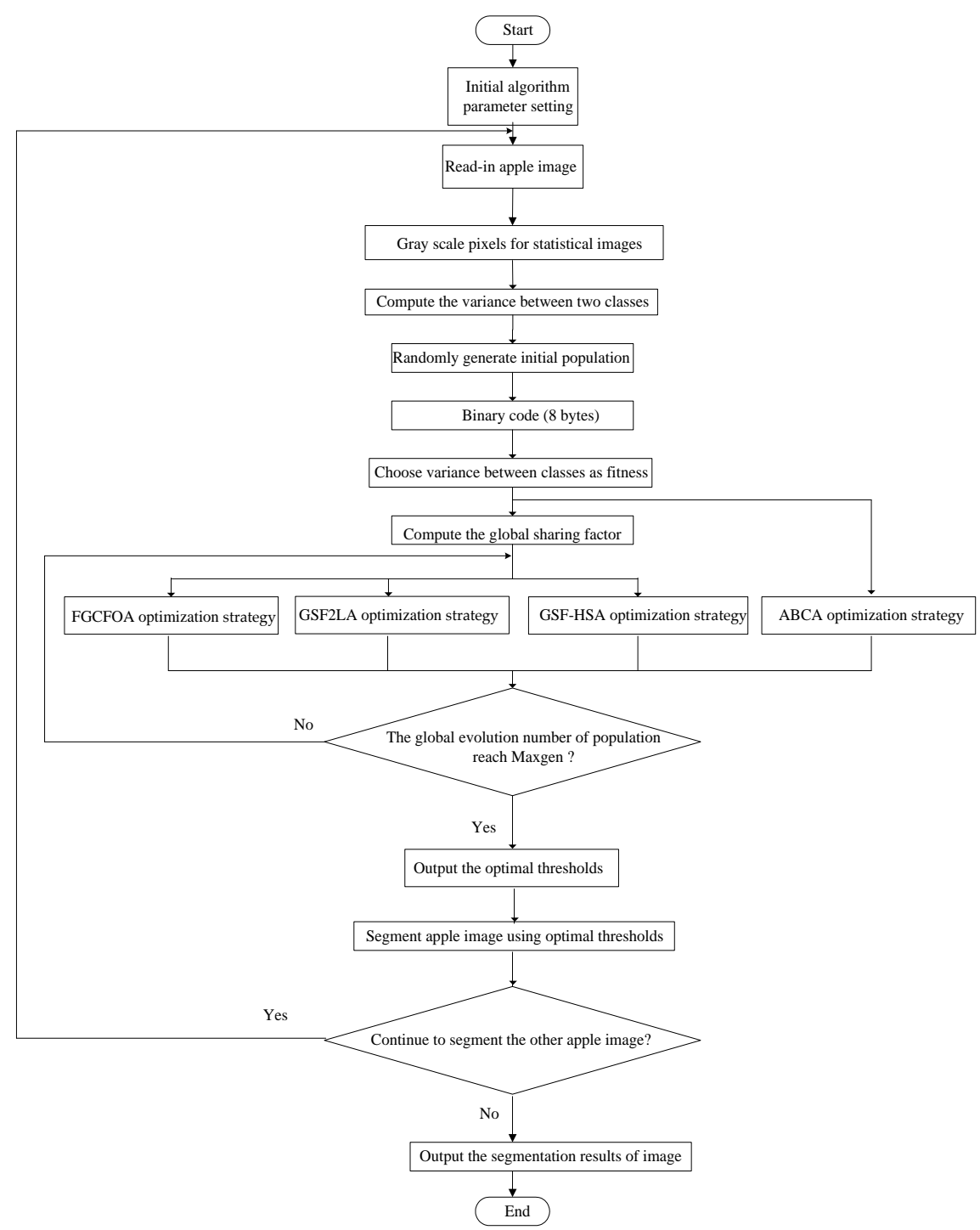

Figure 2. Flow chart of improved threshold segmentation method 


\section{Apple Image Segmentation Model based on R Component}

\subsection{Apple Image Segmentation Model based on R Component with Swarm Intelligence Optimization Algorithm (AISM- RSIOA)}

In this paper, an improved color image cone segmentation method with R component (ISMR) is applied to the segmentation of HuaNiu apple color image, and the swarm intelligence optimization algorithm is introduced to optimize the threshold of image to overcome the defect of over segmentation in the classical threshold segmentation method. As shown in Figure 3, an apple image segmentation model based on R component with swarm intelligence optimization algorithm (AISM-RSIOA) is presented.

In the AISM-RSIOA model, the apple images undergo two rounds of segmentation. Firstly, the model preprocesses the apple image captured in the natural scenes, performs the wavelet transform to remove noises on the image, and then performs median filtering on the image. Then, the model selects the ISMR method to segment the apple image for the first time, which can remove a large number of background elements such as branches and leaves to make the segmentation result clearer. However, after the initial segmentation, there still exist some backgrounds in the apple image which are segmented as the target which makes the segmentation result appear extra noise. To achieve a better segmentation result, the model selects the variance between classes of image segmented by the ISMR method as the fitness of the swarm intelligence algorithm. The segmentation threshold of apple image will be optimized by the four improved swarm intelligence algorithm optimization strategies. Finally, through the second segmentation for the apple image by the improved threshold segmentation method, the range of the $\mathrm{R}$ component is carefully selected to achieve the purpose of eliminating unnecessary pixels, reducing the noise and excess branches in the background.

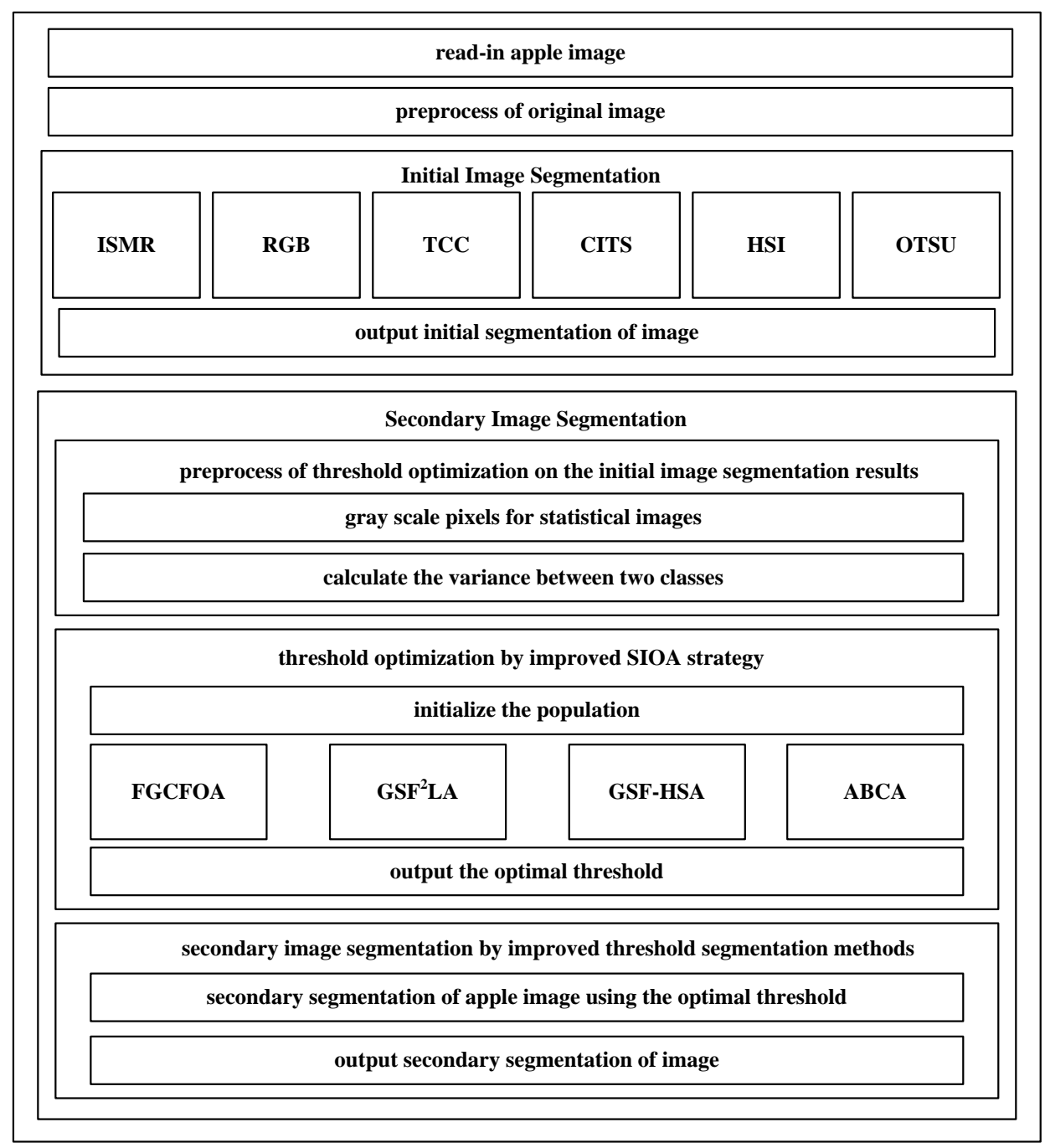

Figure 3. Framework chart of AISM-RSIOA 
The model framework in Figure 3 not only describes how to model the apple image segmentation method under different scenes and different lighting conditions, but also provides a method of horizontal and vertical comparison of the segmentation results of different steps to achieve the purpose of validating the model.

\subsection{Design of AISM-RSIOA Model System}

To make the AISM-RSIOA model easy to use and effective, a graphical user interface (GUI) system for AISM-RSIOA model has been developed base on the Matlab 7.0 software. It also provides an effective comparison platform for the comparative experiments. The model system interface is showed in Figure 4.

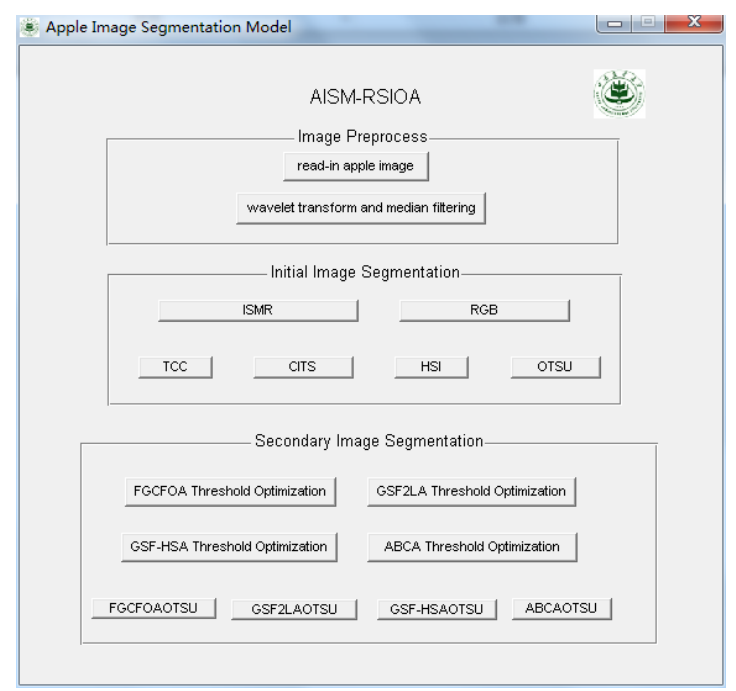

Figure 4. Interface of the AISM-RSIOA system

\section{Experiment Design and Results Analysis}

\subsection{Experiment Design}

The experimental samples were chosen from the mature HuaNiu apples. 30 photos were captured by the camera in the natural scenes of direct sunlight conditions and backlight conditions. A series of mature HuaNiu apple images segmentation experiments are done under six conditions of direct sunlight with strong, medium and weak illumination, and backlighting with strong, medium and weak illumination in natural scene.

The experiments are divided into two steps. Firstly, the initial image segmentation module of AISM-RSIOA model is used to perform the segmentation experiment on six images. Six segmentation methods, such as the improved color image cone segmentation method with R component (ISMR), chromatic aberration method of RGB (RGB), chromatic aberration method of three color component (TCC) [8], color image cone segmentation algorithm (CITS) [27], colorimetric method of HSI (HSI) and classical threshold segmentation method (OTSU) are used to verify the validity of the ISMR method. Secondly, the secondary image segmentation module of AISM-RSIOA model is used to perform the threshold optimization process on the initial image segmentation results, and the secondary image segmentation results are output to verify the validity of the AISM-RSIOA model.

To get a better evaluation of twice segmentation results used by the AISM-RSIOA model, five segmentation indicators based on the image recognition rate in literature [19], such as segmentation rate $\left(\mathrm{R}_{\mathrm{Seg}}\right)$, fruit segmentation rate $\left(\mathrm{R}_{\mathrm{App}}\right)$, branch segmentation rate $\left(\mathrm{R}_{\mathrm{Bra}}\right)$, segmentation success rate $\left(\mathrm{R}_{\mathrm{Suc}}\right)$ and missing rate $\left(\mathrm{R}_{\mathrm{Mis}}\right)$, are respectively defined in this paper. The equations of the five indicators are defined in Equation (12) (16). Among them, $N_{\text {to }}$ is the total number of segmentation targets (including the number of fruits and the number of branches), $N_{p o}$ is the total number of targets obtained by manual visualization (including the number of fruits and the number of branches), $N_{a}$ is the total number of segmentation fruits, $N_{p a}$ is the total number of fruits obtained by manual visualization, $N_{l}$ is the total number of segmentation branches. Due to the effects of illumination condition and interference factors, the experiment set the fruit size that greater than $1 / 4$ 
complete fruit as a fruit statistics value, and set the branch length that greater than $1 / 4$ complete branch as a branch of the statistics value, and too little fruits in the perspective of image are not counted in the statistics value.

$$
\begin{gathered}
R_{\text {Seg }}=\frac{N_{t o}}{N_{p o}} \times 100 \% \\
R_{\text {App }}=\frac{N_{a}}{N_{t o}} \times 100 \% \\
R_{\text {Bra }}=\frac{N_{l}}{N_{t o}}=\frac{N_{t o}-N_{a}}{N_{t o}} \times 100 \% \\
R_{\text {Suc }}=\frac{N_{a}}{N_{p a}} \times 100 \% \\
R_{\text {Mis }}=\frac{N_{p a}-N_{a}}{N_{p a}} \times 100 \%
\end{gathered}
$$

All the algorithms are coded in Matlab 7.0, and the simulations are run on a PC that has an Intel Core2 $2.0 \mathrm{GHz} \mathrm{CPU}$ and 2 GB memory. The average values of 30 times of independent running results are used for the final experiment results.

\subsection{Experiment Results and Analysis}

\subsubsection{Initial Image Segmentation Experiment}

After the image preprocessing, the images are initially segmented by ISMR and other five methods in the AISM-RSIOA model system. The results of the initial segmentation of apple images under the six illumination conditions are compared in Table 1. Figure 5 shows the results of the initial segmentation under the condition of direct sunlight with strong illumination. The figure includes the original image, the pre-processed image and the results of the initial segmentation of the six segmentation methods.

Under the condition of direct sunlight with strong illumination, the segmentation rates of the six segmentation methods are all $100.0 \%$, which shows that all the six segmentation methods take the branches as the segmentation targets. It can also be seen in Figure 5 that there are branches in different degrees in each image after segmentation. This is mainly due to the fact that under the condition of direct sunlight with strong illumination, the color of the branches of the apple is brown and close to red, which is prone to be segmented as the target. However, along with the gradual weakening of the light of direct sunlight, the segmentation rates decreased under conditions of medium illumination and weak illumination, and the fruit segmentation rates increased. This indicates that the degree in which the branches are segmented as a target is reduced. Therefore, the illumination condition has a certain impact on the segmentation results. In the meantime, under the conditions of direct sunlight with medium and weak illumination, the fruit segmentation rates of ISMR are higher than the other five kinds of segmentation methods, and the branch segmentation rate is also lower than other methods. Under the conditions of direct sunlight with weak illumination, the segmentation success rate reaches $90.0 \%$. There exist few missing fruits in segmentation which is mainly because of the dark light.

\subsubsection{Secondary Image Segmentation Experiment}

Improving the accuracy of the segmentation result requires further segmentation using the AISM-RSIOA model to remove the extra background elements. In this paper, the results of images segmented by ISMR are used as the input of the secondary image segmentation experiment. Firstly, the optimal threshold of the image is optimized by the swarm intelligence algorithm in the AISM-RSIOA model system. The optimal threshold of each image is obtained by the four kinds of intelligence algorithms; then, the improved threshold segmentation method is used to segment the image to achieve the purpose of completely separating the fruit from the background.

\section{(1) Swarm Intelligence Algorithm Threshold Optimization Experiment}

In the threshold optimization experiments of the swarm intelligence algorithm, four kinds of swarm intelligence algorithms are used: FGCFOA, GSF²LA, GSF-HSA and ABCA. 
From the perspective of evolutionary convergence speed of swarm intelligence algorithm, FGCFOA has the fastest convergence rate, which is obviously superior to the other three algorithms. The following sequence are ABCA, GSF2LA and GSF-HSA. The thresholds obtained by FGCFOA are the smallest, while the thresholds obtained by GSF-HSA are the largest.

Table 1. Comparison of initial image segmentation results under six illumination conditions

\begin{tabular}{|c|c|c|c|c|c|c|}
\hline Segmentation Method & Illumination Condition & $\mathrm{R}_{\mathrm{Seg}}$ & $\mathrm{R}_{\text {App }}$ & $\mathrm{R}_{\mathrm{Bra}}$ & $\mathrm{R}_{\text {Suc }}$ & $\mathrm{R}_{\mathrm{Mis}}(\%)$ \\
\hline \multirow{6}{*}{ ISMR } & direct sunlight with strong & 100.0 & 75.0 & 25.0 & 100.0 & 0.0 \\
\hline & direct sunlight with medium & 85.7 & 66.7 & 33.3 & 100.0 & 0.0 \\
\hline & direct sunlight with weak & 81.8 & 100.0 & 0.0 & 90.0 & 10.0 \\
\hline & backlighting with strong & 81.8 & 77.8 & 22.2 & 100.0 & 0.0 \\
\hline & backlighting with medium & 90.0 & 100.0 & 0.0 & 100.0 & 0.0 \\
\hline & backlighting with weak & 100.0 & 91.7 & 8.33 & 100.0 & 0.0 \\
\hline \multirow{6}{*}{ RGB } & direct sunlight with strong & 100.0 & 75.0 & 25.0 & 100.0 & 0.0 \\
\hline & direct sunlight with medium & 100.0 & 57.1 & 42.9 & 100.0 & 0.0 \\
\hline & direct sunlight with weak & 81.8 & 88.9 & 11.1 & 80.0 & 20.0 \\
\hline & backlighting with strong & 100.0 & 63.6 & 36.4 & 100.0 & 0.0 \\
\hline & backlighting with medium & 90.0 & 88.9 & 11.1 & 88.9 & 11.1 \\
\hline & backlighting with weak & 91.7 & 90.9 & 9.1 & 90.9 & 9.1 \\
\hline \multirow{6}{*}{ TCC } & direct sunlight with strong & 100.0 & 75.0 & 25.0 & 100.0 & 0.0 \\
\hline & direct sunlight with medium & 100.0 & 57.1 & 42.9 & 100.0 & 0.0 \\
\hline & direct sunlight with weak & 81.8 & 88.9 & 11.1 & 80.0 & 20.0 \\
\hline & backlighting with strong & 81.8 & 77.8 & 22.2 & 100.0 & 0.0 \\
\hline & backlighting with medium & 90.0 & 88.9 & 11.1 & 88.9 & 11.1 \\
\hline & backlighting with weak & 91.7 & 90.9 & 9.1 & 90.9 & 9.1 \\
\hline \multirow{6}{*}{ CITS } & direct sunlight with strong & 100.0 & 75.0 & 25.0 & 100.0 & 0.0 \\
\hline & direct sunlight with medium & 100.0 & 57.1 & 42.9 & 100.0 & 0.0 \\
\hline & direct sunlight with weak & 63.6 & 100.0 & 0.0 & 70.0 & 30.0 \\
\hline & backlighting with strong & 100.0 & 63.6 & 36.4 & 100.0 & 0.0 \\
\hline & backlighting with medium & 90.0 & 88.9 & 11.1 & 88.9 & 11.1 \\
\hline & backlighting with weak & 66.7 & 87.5 & 12.5 & 63.6 & 36.4 \\
\hline \multirow{6}{*}{ HSI } & direct sunlight with strong & 100.0 & 75.0 & 25.0 & 100.0 & 0.0 \\
\hline & direct sunlight with medium & 100.0 & 57.1 & 42.9 & 100.0 & 0.0 \\
\hline & direct sunlight with weak & 81.8 & 100.0 & 0.0 & 90.0 & 10.0 \\
\hline & backlighting with strong & 100.0 & 63.6 & 36.4 & 100.0 & 0.0 \\
\hline & backlighting with medium & 60.0 & 100.0 & 0.0 & 66.7 & 33.3 \\
\hline & backlighting with weak & 58.3 & 100.0 & 0.0 & 63.6 & 36.4 \\
\hline \multirow{6}{*}{ OTSU } & direct sunlight with strong & 75.0 & 66.7 & 33.3 & 66.7 & 33.3 \\
\hline & direct sunlight with medium & 100.0 & 57.1 & 42.9 & 100.0 & 0.0 \\
\hline & direct sunlight with weak & 63.6 & 85.7 & 14.3 & 60.0 & 40.0 \\
\hline & backlighting with strong & 100.0 & 63.6 & 36.4 & 100.0 & 0.0 \\
\hline & backlighting with medium & 70.0 & 85.7 & 14.3 & 66.7 & 33.3 \\
\hline & backlighting with weak & 66.7 & 87.5 & 12.5 & 63.6 & 36.4 \\
\hline
\end{tabular}




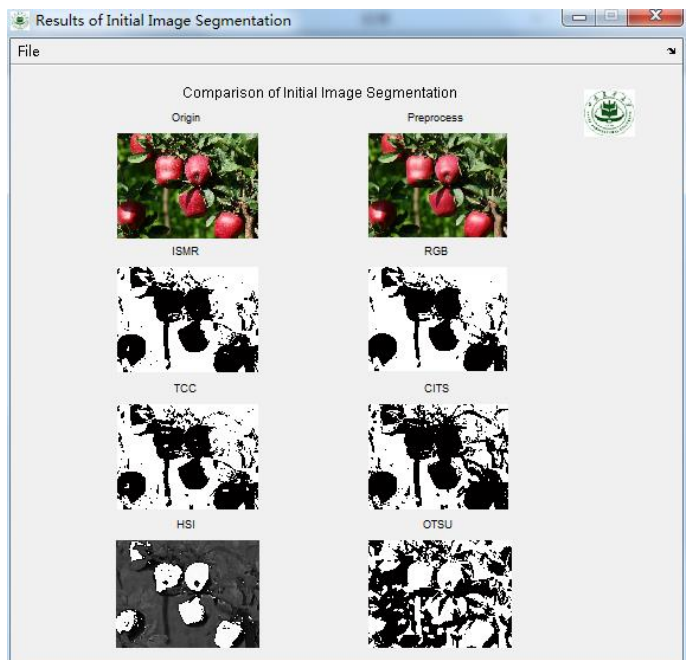

Figure 5. Initial segmentation results of apple image under direct sunlight with strong illumination condition

Table 2. Comparison of secondary image segmentation effect under six illumination conditions

\begin{tabular}{|c|c|c|c|c|c|c|}
\hline Segmentation Method & Illumination Condition & $\mathrm{R}_{\mathrm{Seg}}$ & $\mathrm{R}_{\mathrm{App}}$ & $\mathrm{R}_{\mathrm{Bra}}$ & $\mathrm{R}_{\mathrm{Suc}}$ & $\mathrm{R}_{\mathrm{Mis}}(\%)$ \\
\hline \multirow{6}{*}{$\begin{array}{l}\text { FGCFOA } \\
\text { OTSU }\end{array}$} & direct sunlight with strong & 87.5 & 85.7 & 14.3 & 100.0 & 0.0 \\
\hline & direct sunlight with medium & 57.1 & 100.0 & 0.0 & 100.0 & 0.0 \\
\hline & direct sunlight with weak & 81.8 & 100.0 & 0.0 & 90.0 & 10.0 \\
\hline & backlighting with strong & 63.6 & 100.0 & 0.0 & 100.0 & 0.0 \\
\hline & backlighting with medium & 90.0 & 100.0 & 0.0 & 100.0 & 0.0 \\
\hline & backlighting with weak & 91.7 & 100.0 & 0.0 & 100.0 & 0.0 \\
\hline \multirow{6}{*}{$\begin{array}{l}\text { GSF}^{2} L A \\
\text { OTSU }\end{array}$} & direct sunlight with strong & 62.5 & 100.0 & 0.0 & 83.3 & 16.7 \\
\hline & direct sunlight with medium & 42.9 & 100.0 & 0.0 & 75.0 & 25.0 \\
\hline & direct sunlight with weak & 72.7 & 100.0 & 0.0 & 80.0 & 20.0 \\
\hline & backlighting with strong & 45.5 & 100.0 & 0.0 & 71.4 & 28.6 \\
\hline & backlighting with medium & 70.0 & 100.0 & 0.0 & 77.8 & 22.2 \\
\hline & backlighting with weak & 41.7 & 100.0 & 0.0 & 45.5 & 54.5 \\
\hline \multirow{6}{*}{$\begin{array}{l}\text { GSF-HSA } \\
\text { OTSU }\end{array}$} & direct sunlight with strong & 62.5 & 100.0 & 0.0 & 83.3 & 16.7 \\
\hline & direct sunlight with medium & 28.6 & 100.0 & 0.0 & 50.0 & 50.0 \\
\hline & direct sunlight with weak & 63.6 & 100.0 & 0.0 & 70.0 & 30.0 \\
\hline & backlighting with strong & 18.2 & 100.0 & 0.0 & 28.6 & 71.4 \\
\hline & backlighting with medium & 50.0 & 100.0 & 0.0 & 55.6 & 44.4 \\
\hline & backlighting with weak & 25.0 & 100.0 & 0.0 & 27.3 & 72.7 \\
\hline \multirow{6}{*}{$\begin{array}{l}\text { ABCA } \\
\text { OTSU }\end{array}$} & direct sunlight with strong & 62.5 & 100.0 & 0.0 & 83.3 & 16.7 \\
\hline & direct sunlight with medium & 42.9 & 100.0 & 0.0 & 75.0 & 25.0 \\
\hline & direct sunlight with weak & 72.7 & 100.0 & 0.0 & 80.0 & 20.0 \\
\hline & backlighting with strong & 45.5 & 100.0 & 0.0 & 71.4 & 28.6 \\
\hline & backlighting with medium & 80.0 & 100.0 & 0.0 & 88.9 & 11.1 \\
\hline & backlighting with weak & 58.3 & 100.0 & 0.0 & 63.6 & 36.4 \\
\hline
\end{tabular}

\section{(2) Improved Threshold Segmentation Experiment}

The optimized image thresholds and the image segmentation results obtained from the ISMR method are taken for the secondary image segmentation experiment. Then, the improved threshold segmentation methods of FGCFOAOTSU, 
GSF²LAOTSU, GSF-HSAOTSU and ABCAOTSU are used for the experiments, respectively. The secondary segmentation results are shown in Table 2, and Figure 6 is the result of secondary segmentation under the condition of direct sunlight with strong illumination. The figure includes the original image, the result image segmented by the ISMR method, and the results of four improved threshold segmentation methods and the threshold.

In Table 2, under the condition of direct sunlight with strong illumination, the fruit segmentation rate of FGCFOAOTSU is $85.7 \%$. Under other illumination conditions, the fruit segmentation rates all reach $100.0 \%$ and are greatly improved compared with the initial segmentation result. It also shows that the four methods have a branch segmentation rate of $0.0 \%$ in Table 2, which indicates that the background factors such as branches can be removed better in the secondary segmentation. In addition, the segmentation success rate of FGCFOAOTSU is $90.0 \%$ under the condition of direct sunlight with weak illumination, and it can reach $100.0 \%$ under all other conditions.

As can be seen in Figure 6, the secondary segmentation can remove the background interference factors well. The four improved threshold segmentation methods can completely separate the fruit from the background. Compared with the initial segmentation method of ISMR, there are significant declines in the segmentation rates. It also can be found in the figures that the thresholds are closely related to the effects of the secondary segmentation. The smaller of the threshold, the higher the segmentation success rate of the improved threshold segmentation method, whereas there will be over segmentation which can seriously damage the segmentation effect. The success rates of the other three methods in Table 2 are lower than the FGCFOAOTSU method, and the FGCFOAOTSU method has the better image segmentation result.

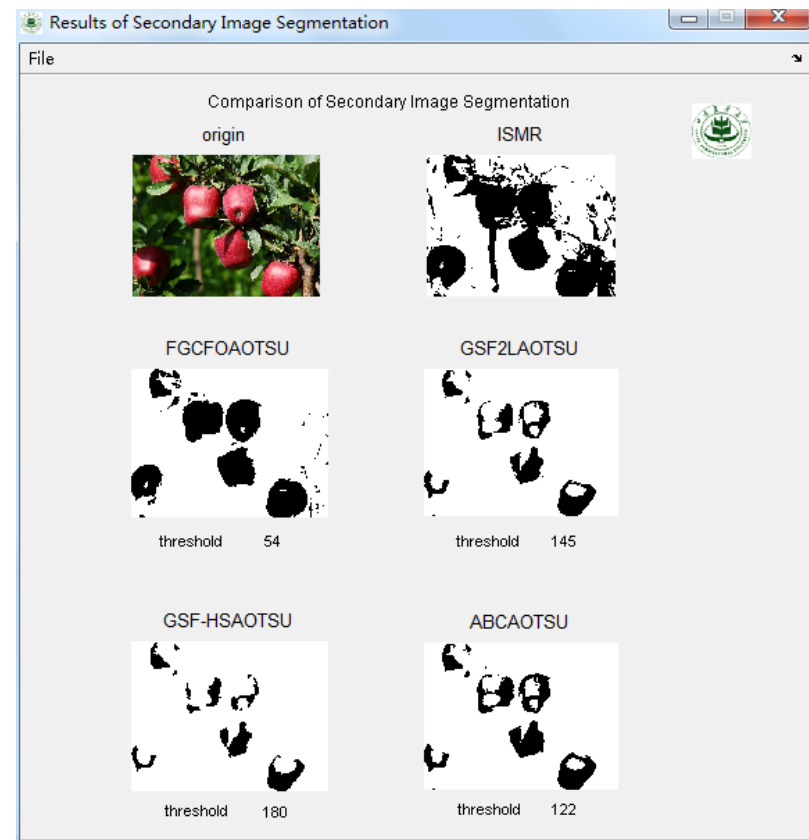

Figure 6. Secondary segmentation results of apple image under direct sunlight with strong illumination condition

\section{Conclusions}

Because of large numbers of interference factors and the complex background in apple images in natural scenes, it is difficult to achieve good segmentation results. To solve this problem, the color apple image segmentation method under natural scenes is modeled to construct a unified segmentation model (AISM-RSIOA) in this paper. The experiment is designed to segment the photos of mature HuaNiu apples under six illumination conditions in natural scene. The experimental results show that the ISMR method of AISM-RSIOA model has the best initial segmentation results, and the background factors such as branches can be removed effectively in the secondary segmentation. The experimental results validated that the model is effective and feasible, and the model system is convenient and easy to operate. Future work is to research the optimization methods of apple images based on the multi-objective swarm intelligence algorithm.

\section{Acknowledgements}

This work is supported by the National Nature Science Foundation of China (Grant No. 61741201, 61462058). 


\section{References}

1. X. A. Bao, R. L. Zhang, L. H. Zhong, "Apple Grade Identification Method based on Artificial Neural Network and Image Processing," Transactions of the Chinese Society of Agricultural Engineering, vol. 20, no. 3, pp. 109-112, 2004

2. X. M. Bao, Y. M. Wang, "Apple Image Segmentation based on the Minimum Error Bayes Decision." Transactions of the Chinese Society of Agricultural Engineering, vol. 22, no. 5, pp. 122-124, 2016

3. D. M. Bulanon, T. Kataoka, Y. Ota, "AE-Automation and Emerging Technologies: A Segmentation Algorithm for the Automatic Recognition of Fuji Apples at Harvest," Biosystems Engineering, vol. 83, no 4, pp. 405-412, 2002

4. M. M. Eusuff, K. E. Lansey, "Optimization of Water Distribution Network Design Using the Shuffled Frog Leaping Algorithm," Journal of Water Sources Planning and Management, vol. 129, no. 3, pp. 210- 225, 2003

5. Z. W. Geem, J. H. Kim, G. V. Loganathan, "A New Heuristic Optimization Algorithm: Harmony Search," Simulation, vol. 76, no. 2, pp. 60-68, 2001

6. J. Y. Han, C. Z. Liu, L G. Wang, "Dynamic Double Subgroups Cooperative Fruit Fly Optimization Algorithm," Pattern Recognition and Artificial Intelligence, vol. 26, no. 11, pp. 1057-1067, 2013

7. M. Hu, M. Li, R. G. Wang, "Application of an Improved OTSU Algorithm in Image Segmentation," Journal of Electronic Measurement and Instrument, vol. 24, no. 5, pp. 443-449, 2010

8. M. D. Hu, L. J. Yang, S. D. Zhu, "Traffic Sign Segment based on Chromatic Aberration of Three Color Components," Mechanical \& Electrical Engineering Magazine, vol. 26, no. 10, pp. 23-26, 2009

9. Y. S. Jiang, X. L. Song, J. J. Ren, "2D OTSU Algorithm Improvement based on Genetic Algorithm," Application Research of Computers, vol. 27, no. 3, pp. 1189-1191, 2010

10. D. Karaboga, "An Idea Based on Honey Bee Swarm for Numerical Optimization," Erciyes University, Technical Report: TR06, pp. 6-16, 2005

11. J. F. Kennedy, R. C. Eberhart, Y. Shi, "Swarm Intelligence," Morgan Kaufmann Publishers, San Francisco, Calif, USA, pp. 101-109, 2001

12. H. G. Li, L. Yao, L. Shi, "Automatic Selection of Image Threshold based on Improved OTSU," Computer simulation, vol. 24, no. 4, pp. 216-220, 2007

13. C. Z. Liu, J. Y. Han, "Adaptive Fruit Fly Optimization Algorithm based on Bacterial Migration," Computer Engineering \& Science, vol. 36, no. 4, pp. 690-696, 2014

14. L. Q. Liu, J. Y. Huo, L. G. Wang, "Harmony Search Algorithm with Global Sharing Factor," Journal of Chongqing University of Technology (Natural Science), vol. 28, no. 2, pp. 82-86, 2014

15. L. Q. Liu, L. G. Wang, J. Y. Han, "Shuffled Frog Leaping Algorithm based on Global Sharing Factor," Computer Engineering, vol. 39, no. 10, pp. 162-166, 2013

16. B. B. Lu, Z. H. Jia, D. He, "Remote-sensing Image Segmentation Method based on Improved OTSU and Shuffled Frog-Leaping Algorithm," Computer Applications and Software, vol. 28, no. 9, pp. 77-79, 2011

17. N. OTSU, "A Threshold Selection Method from Gray-Level Histograms," IEEE Transactions on Systems, Man, and Cybernetics, vol. 9, no. 1, pp. 62-66, 1979

18. W. T. Pan, "A New Fruit Fly Optimization Algorithm: Taking the Financial Distress Model as an Example," Knowledge-Based Systems, vol. 29, pp. 69-74, 2012

19. J. P. Qian, X. T. Yang, X. M.Wu, "Mature Apple Recognition based on Hybrid Color Space in Natural Scene," Transactions of the Chinese Society of Agricultural Engineering, vol. 28, no. 17, pp. 137-142, 2012

20. P. K. Shukla, K. Deb, "On Finding Multiple Pareto-Optimal Solutions Using Classical and Evolutionary Generating," European Journal of Operational Research, vol. 181, no. 3, pp.1630-1652, 2007

21. Y. S. Si, J. Qiao, G. Liu, "Recognition and Shape Features Extraction of Apples based on Machine Vision," Transactions of the Chinese Society of Agricultural Machinery, vol. 40, no. 8, pp. 161-165, 2009

22. A. L. Tabb, D. L. Peterson, J. Park, "Segmentation of Apple Fruit from Video via Background Modeling," ASABE Meeting, 2006

23. J. Tu, C. L. Liu, Y. M. Li, "Apple Recognition Method based on Illumination Invariant Graph," Transactions of the Chinese Society of Agricultural Engineering, vol. 26, no. 2, pp. 26-31, 2010

24. H. Wang, "Artificial Bee Colony Algorithm with Sharing Factor," Computer Engineering, vol. 37, no. 22, pp. 139-142, 2011

25. J. J. Wang, D. A. Zhao, W. Ji, "Apple Fruit Recognition based on Support Vector Machine Using in Harvesting Robot," Transactions of the Chinese Society of Agricultural Machinery, vol. 40, no. 1, pp. 148-151, 2009

26. Y. J. Zhang, M. Z. Li, J. Qiao, "Segmentation Algorithm for Apple Recognition Using Image Features and Artificial Neural Network," Acta Optica Sinica, vol. 28, no. 11, pp. 2104-2108, 2008

27. B. Zheng, "Agricultural Image Processing System Design and Algorithm Research based on Cloud Computing," Jiangsu: Jiangsu University, vol. 12, pp. 12:55-83, 2012

Liqun Liu is an associate professor at the College of Information Science and Technology, Gansu Agricultural University. Her research interests include the swarm intelligence algorithm.

Jiuyuan Huo is a professor at the Lanzhou Jiaotong University. He received his doctoral degree from Chinese Academy of Sciences in 2012. His research interests include optimization algorithms and data mining. 